PRAXIS

ecucativa

Universidad Nacional de La Pampa

Facultad de Ciencias Humanas

Instituto de Ciencias de la Educación

para la investigación interdisciplinaria

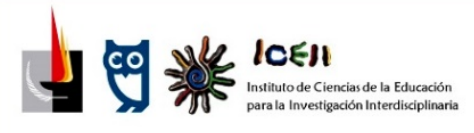

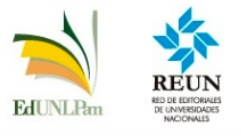

ISSN 2313-934X

SANTA ROSA, LA PAMPA, ARGENTINA

Correo electrónico: iceii@humanas.unlpam.edu.ar

Disponible en https://cerac.unlpam.edu.ar/index.php/praxis

Formación docente con inclusión de medios digitales. La experiencia del área de Ciencias Sociales del plan Escuelas de Innovación (20112015) desde las voces de sus protagonistas. Artículo de Braian Marchetti y Sonia Bazán. Praxis educativa, Vol. 26, No 1 enero - abril 2022. E - ISSN 2313-934X. pp. 1-20. https://dx.doi.org/10.19137/praxiseducativa-2022-260117

Esta obra se publica bajo Licencia Creative Commons 4.0 Internacional CC BY-NC-SA Atribución, No Comercial, Compartir igual

\title{
Formación docente con inclusión de medios digitales. La experiencia del área de Ciencias Sociales del plan Escuelas de Innovación (2011- 2015) desde las voces de sus protagonistas
}

Teacher training with digital media inclusion. The experience in the Social Sciences area of the Escuelas de Innovación program (2011-2015) from its protagonists' voices

Formação de professores incluindo mídia digital. A experiência da área de Ciências Sociais no plano Escolas de Inovação (2011-2015) a partir das vozes dos seus protagonistas

\section{Braian Marchetti}

Universidad Nacional de Mar del Plata, Consejo Nacional de Ciencia y Tecnología CONICET, Argentina braian.marchetti@hotmail.com

ORCID 0000-0002-7207-5031

\section{Sonia Bazán}

Universidad Nacional de Mar del Plata, Argentina

bazansa@gmail.com

ORCID 0000-0003-0465-4303

Recibido: 2021-11-01 | Revisado: 2021-12-08 | Aceptado: 2021-12-14 


\section{Resumen}

Las políticas públicas de formación docente destinadas a la inclusión digital han tomado centralidad al calor de las transformaciones sociotécnicas y las situaciones surgidas en relación con la conectividad y el uso de medios digitales en educación a partir de las medidas de aislamiento que se tomaron para enfrentar al Covid-19. Consideramos de relevancia recuperar experiencias formativas pasadas que tuvieron lugar durante el desarrollo del programa de mayor envergadura en la materia como lo fue Conectar Igualdad, a fin de aportar a la reflexión y planificación de futuras y necesarias políticas formativas.

Desde los hallazgos de una investigación mayor realizada sobre las políticas públicas de formación docente con inclusión de medios digitales, en la cual hemos profundizado en el caso del plan Escuelas de Innovación (2011-2015), en este trabajo analizamos el recorrido del equipo de capacitación del área de Ciencias Sociales de este plan desde las voces de sus integrantes y de docentes participantes de las capacitaciones.

Palabras clave: formación docente - inclusión digital - Ciencias Sociales - Escuelas de Innovación - Conectar Igualdad

\section{Abstract}

Public policies in teacher training devoted to digital inclusion have become relevant in the context of sociotechnical transformations and situations related to connectivity and the use of digital media in education since the lockdown for Covid-19. In this scenario, it is essential to look into past experiences which took place during the development of the most important national program: Conectar lgualdad, in order to reflect upon and plan future formative policies.

From the findings of a broader research which centered on public policies of teacher training with digital inclusion and the case of the Escuelas de Innovación program (2011-2015), this work analyzes the paths of the training team in the Social Sciences area from the voices of its members and those of the teachers participating in such training.

Keywords: teacher training - digital inclusion - Social Sciences - Escuelas de Innovación - Conectar Igualdad

\section{Resumo}

As políticas públicas de formação de professores voltadas à inclusão digital têm assumido centralidade em meio às transformações sociotécnicas e às situações que surgiram em relação à conectividade e ao uso das mídias digitais na educação a partir das medidas de isolamento que foram tomadas para enfrentar a Covid19. Consideramos importante resgatar experiências de formação anteriores ocorridas durante o desenvolvimento do maior programa na área, como o "Conectar Igualdad", a fim de contribuir para a reflexão e o planejamento de futuras políticas de formação.

A partir dos resultados de uma importante pesquisa realizada sobre políticas públicas de formação de professores com inclusão de mídias digitais, na qual nos aprofundamos no caso do plano Escolas de Inovação (2011-2015), analisamos neste trabalho o percurso da equipe formadora na área de Ciências Sociais deste plano a partir da voz dos seus membros e docentes participantes nas formações.

Palavras-chave: formação de professores - inclusão digital - Ciências Sociais - Escolas de inovação 


\section{Introducción}

Durante las últimas dos décadas y, fundamentalmente, luego de atravesada la pandemia durante los últimos dos años, se ha puesto en evidencia la relevancia y la necesidad de las políticas de inclusión digital destinadas al ámbito educativo. En Argentina, la iniciativa de mayor envergadura en la materia fue el lanzamiento del Programa Conectar Igualdad ( $\mathrm{PCl}$ ) en el año 2010 cuyas netbooks, aún más de una década después, siguen presentes en el uso de estudiantes y docentes. Al momento de su creación, el PCl generó la irrupción en el debate público sobre las temáticas referidas a la conectividad, al derecho a la alfabetización digital y a la necesidad de intervención estatal para garantizar que todos los estudiantes y docentes puedan acceder a las tecnologías.

La creación del PCl y la distribución de más de cinco millones de netbooks le otorgó un fuerte impulso también al desarrollo de políticas y acciones dirigidas a la formación docente en pos de la integración de los medios digitales en las propuestas educativas. Tanto para la formación inicial como para la formación en ejercicio, desde el ámbito privado y fuertemente desde el ámbito público tuvieron lugar una serie de planes y programas formativos que tuvieron como objetivo la construcción de abordajes didácticos con el uso de medios digitales.

Las políticas públicas de inclusión digital se convirtieron así en una preocupación y también en tema de investigación desarrollado por equipos de distintas universidades de nuestro país. Los estudios realizados por el Grupo de Investigaciones en Didáctica de la Historia y las Ciencias Sociales (CIEDHICS) al cual pertenecemos, junto con el Centro de Investigaciones Multidisciplinarias en Educación (CIMED) de la Facultad de Humanidades de la Universidad Nacional de Mar del Plata (FH/UNMDP), muestran preocupaciones comunes y análisis particulares acerca de la formación docente en tiempos de inmersión tecnológica. Desde este marco institucional, hemos llevado adelante una investigación mayor ${ }^{1}$ a este artículo en la cual indagamos sobre las políticas públicas de formación docente en ejercicio con inclusión de medios digitales. Para ello tomamos como caso de análisis la experiencia del plan de capacitación Escuelas de Innovación (EI) y su propuesta para la enseñanza de las Ciencias Sociales. Este plan fue creado en el año 2011 desde ANSES y formó parte de las iniciativas dirigidas a docentes y equipos directivos que acompañaron la entrega de netbooks del $\mathrm{PCl}$.

Desde una metodología cualitativa con un enfoque narrativo, en nuestra investigación nos abocamos a la recuperación de las voces de los sujetos que protagonizaron las políticas públicas aludidas anteriormente, a fin de interpretar el alcance de las propuestas de formación docente en inclusión de medios digitales en el área de Ciencias Sociales del plan Escuelas de Innovación. Para ello realizamos una serie de entrevistas en profundidad a quienes formaron parte de los equipos de gestión del Ministerio de Educación de la Nación, del PCl, de El y de la gestión educativa de la provincia de Mendoza. Además, recuperamos las voces de docentes de esa provincia que formaron parte de la propuesta de capacitación de El.

A partir de la relevancia otorgada a las propuestas formativas de inclusión digital, y desde el convencimiento que es necesario profundizar el camino iniciado hace algunos años en materia 
Formación docente con inclusión de medios digitales. La experiencia del área de Ciencias Sociales del plan Escuelas de Innovación (2011-

2015) desde las voces de sus protagonistas. |

Braian Marchetti y Sonia Bazán.

de políticas de formación docente en ejercicio, en este artículo recuperamos la experiencia de conformación y trabajo del área de Ciencias Sociales de El. Es por ello que realizamos un recorrido por la dinámica que tuvo el proceso de conformación inicial del grupo de capacitadores, por las tareas de capacitación que estuvieron a cargo del área de Ciencias Sociales, por la dinámica de trabajo que adoptó para llevar estas tareas adelante, así como por las dificultades que se le presentaron y las opiniones de los docentes que participaron de las capacitaciones. Consideramos que retomar el trabajo realizado por El en el marco del PCI nos permite construir un insumo para reflexionar sobre el diseño y desarrollo de próximas políticas formativas.

\section{El plan Escuelas de Innovación y su propuesta formativa}

Generar políticas públicas que permitan romper con las brechas de acceso y de uso a las tecnologías deviene en una responsabilidad del Estado. Una de las experiencias más importantes que da cuenta de la atención del Estado argentino al respecto, ha sido el Programa Conectar Igualdad (PCI). Este programa fue lanzado en el año 2010 por decreto presidencial № 459/10 como acción de política pública para la inclusión digital educativa destinada a garantizar el acceso y uso de las tecnologías a estudiantes y docentes de distintos niveles del sistema educativo. Hacia agosto de 2015 el PCl había entregado más de 5 millones de netbooks alcanzando los 11.000 establecimientos educativos e incidiendo positivamente en la disminución de la brecha digital (Ministerio de Educación de la Nación, 2015).

El PCl además de realizar la entrega de netbooks y de brindar soporte informático y técnico a los establecimientos educativos, incluyó dentro de su propuesta a la formación docente. Su lanzamiento dio lugar a la proliferación de distintas instancias formativas que consistieron desde encuentros y reuniones informativas para brindar una primera aproximación al programa, a la formación directa de docentes a través de cursos, seminarios, talleres, jornadas institucionales, seminarios virtuales y la elaboración de secuencias didácticas, bajo el objetivo de ofrecer a los docentes la posibilidad de la incorporación de conceptos, herramientas y propuestas de trabajo vinculadas a la integración de las tecnologías en el ámbito escolar. Al mismo tiempo, se realizaron acciones destinadas a equipos directivos y otros actores de las instituciones orientadas hacia el acompañamiento de la gestión institucional y la organización de los equipos docentes.

Dentro de las primeras acciones surgidas desde el nivel central del Ministerio de Educación es posible identificar a aquellas dirigidas a realizar una sensibilización o primera aproximación a todos los supervisores y directivos de las escuelas que iban siendo alcanzadas en jornadas presenciales de dos días. Luego, se emprendieron para la educación secundaria un grupo de acciones de formación nacionales destinadas a supervisores, equipos de conducción y a formadores disciplinares. Asimismo, se desarrollaron en los primeros años más de 100 cursos virtuales autoasistidos y otros moderados ejecutados por Educ.ar. Junto con estas iniciativas tuvieron lugar también las formulaciones realizadas por las jurisdicciones que implicaron la construcción situada de las formas de intervención, en el marco de los lineamientos concertados federalmente y los escenarios particulares que fue necesario atender. Con sus diferentes formatos 
y énfasis, las iniciativas se destinaron principalmente a la sensibilización en políticas de inclusión digital y modelo uno a uno, la alfabetización y/o profundización en saberes digitales para los distintos actores escolares y otras acciones que pueden involucrar iniciativas dirigidas al seguimiento, evaluación y sistematización de experiencias, al trabajo con estudiantes y familias, entre otras (Perczyck et al., 2011).

De esta manera, dentro de las líneas de formación docente en ejercicio para el período estudiado, convivieron una serie de propuestas a nivel nacional y local articuladas por el Ministerio de Educación de la Nación y los Institutos de Formación Docente públicos y privados, con iniciativas propias de las jurisdicciones provinciales, de las universidades nacionales, fundaciones y empresas privadas. A fines de nuestro interés en la propuesta estatal es posible reconocer distintas líneas desarrolladas específicamente para la formación en ejercicio. En ese marco, uno de los planes que, radicado en la ANSES, tuvo lugar entre los años 2011 y 2015 fue Escuelas de Innovación (El).

Dentro de las premisas principales del plan estuvieron dar lugar a la incorporación de tecnologías en las prácticas de enseñanza del nivel secundario, articular sus acciones principalmente en la realización de capacitaciones de tipo presencial para docentes y directivos de todo el país, desarrollar espacios de intercambio virtual, y documentar la experiencia realizada. El plan tuvo además dentro de su propuesta dos líneas de trabajo a destacar en relación con el desarrollo de perspectivas de gestión institucional para la inclusión de tecnologías y con el abordaje de la transformación de las prácticas de enseñanza a partir de los contenidos de las distintas áreas disciplinares (ANSES, 2015). Sólo entre 2013 y 2015 desde Escuelas de Innovación se realizaron más de mil encuentros de capacitación en escuelas de distintas localidades de las provincias de Buenos Aires (50 jurisdicciones), Catamarca, Entre Ríos, Mendoza, Misiones, San Juan, Santa Cruz, Tucumán y Jujuy. Estos encuentros presenciales eran acordados y organizados con las distintas jurisdicciones provinciales y se planificaban de manera cuatrimestral.

La organización interna del plan se articuló a través de tres grandes áreas de trabajo compuestas por el Área de Gestión Educativa, el Área Disciplinar y el Área Transversal. La primera, responsable de la transmisión de experiencias y el acompañamiento a supervisores y directores en los temas y herramientas claves para la incorporación de las tecnologías en la gestión institucional de las escuelas. El Área Disciplinar, tuvo a cargo la tarea de brindar asesoramiento para la incorporación de las tecnologías en las propuestas de enseñanza de las áreas de Ciencias Naturales, Ciencias Sociales, Matemática y Lengua y Literatura, mediante un esquema de funcionamiento donde cada área específica contaba con una coordinadora y un grupo de capacitadores que conformaban el equipo de trabajo disciplinar. La tercera área, el Área Transversal, estuvo a cargo de acompañar el desarrollo de propuestas de inclusión digital que implicaran desafíos transdisciplinares, tanto en contenidos de Competencias Digitales y Educación Ambiental como en aspectos vinculados a la modalidad de Educación Especial. Este esquema organizativo a su vez, se orientaba hacia una idea de trabajo sustentable al pretender que la propuesta perdure una vez terminada la intervención presencial, replicable, a fin de que los 
actores llevaran adelante la propuesta en otras escuelas de la jurisdicción, y escalable, para que la propuesta pueda extenderse a un mayor número de jurisdicciones y aumente la cantidad de capacitaciones realizadas (ANSES, 2015a).

A partir de nuestra pertenencia institucional al GIEDHICS y nuestra formación previa en el Profesorado en Historia, seleccionamos al área disciplinar de las Ciencias Sociales de Escuelas de Innovación como el objeto de estudio que nos permitiera profundizar sobre las propuestas generadas por el plan para llevar al aula junto con sus desafíos, problemáticas y potencialidades. En un trabajo anterior (Marchetti y Bazán, 2019), hemos descripto las posibilidades que los medios digitales ofrecen para la enseñanza de las Ciencias Sociales, así como la importancia y centralidad de la tarea de mediación del docente en la construcción de propuestas de enseñanza con tecnologías.

Soletic (2015) señala que para las disciplinas sociales en particular, los medios digitales abren posibilidades para explorar el mundo social en la web e investigarlo, promover los procesos de escritura, crítica y reescritura, analizar imágenes e intervenirlas, organizar la información y representarla visualmente, producir relatos apelando a los lenguajes audiovisuales, analizar la dimensión espacial de las relaciones sociales a través de cartografía dinámica y herramientas de georreferenciación, y/o diseñar narrativas digitales para el análisis de casos y problemas. Ahora bien, estas posibilidades que abre la inclusión de tecnologías no constituyen un fin en sí mismo, sino que las entendemos desde un lugar de mediación dentro de una propuesta más amplia que permita articular los contenidos con los objetivos de enseñanza presentados a través de propuestas didácticas que incluyan el trabajo con tecnologías.

Desde este marco, consideramos que el desafío de las propuestas de formación docente para la enseñanza de las Ciencias Sociales posee por delante la necesidad de formar a los docentes para que sean capaces de articular los objetivos de enseñanza con las potencialidades que los medios digitales posibilitan. Por lo tanto, recuperar la experiencia de construcción y trabajo del dispositivo formador de Escuelas de Innovación para la enseñanza de Ciencias Sociales resulta de interés a fin de ampliar los debates en torno a la inclusión de tecnologías en educación.

\section{Aspectos metodológicos}

El presente trabajo forma parte de una investigación mayor en la que nos hemos propuesto indagar sobre el alcance de las políticas públicas nacionales de formación en ejercicio con inclusión de medios digitales para la enseñanza de las Ciencias Sociales, a través de un análisis del Plan de capacitación docente Escuelas de Innovación (2011-2015). Este estudio nos permitió recuperar la experiencia del proceso inédito que generó el PCI para la formación docente e indagar sobre el desarrollo de estas políticas a nivel nacional y provincial.

La investigación se define como cualitativa e interpretativa (Denzin y Lincoln, 2011) y su desarrollo metodológico consistió principalmente en el análisis documental (Rapley, 2014), y en la realización de entrevistas en profundidad (Robles, 2011). Las voces y prácticas de los sujetos que intervinieron en los procesos de diseño y puesta en acto de Escuelas de Innovación fueron 
abordadas desde el enfoque narrativo (Bolívar, Domínguez y Fernández, 2001; Porta y Flores, 2017). En las entrevistas en profundidad a decisores de políticas educativas, capacitadores de Escuelas de Innovación y docentes capacitados, se buscó interpretar dicha experiencia de política de formación en ejercicio desde su planificación y diseño hasta su puesta en acto en los establecimientos educativos. Estas entrevistas fueron llevadas a cabo en la Ciudad Autónoma de Buenos Aires y la ciudad de Mendoza en cinco etapas, entre noviembre de 2017 y julio de 2019.

En las entrevistas realizadas a los capacitadores de Escuelas de Innovación les solicitamos que nos indicaran cuál fue según su criterio la jurisdicción en donde el desarrollo de la propuesta del plan tuvo mejor funcionamiento y mayor potencia en sus resultados. Los capacitadores señalaron de manera unánime a la provincia de Mendoza, por lo que la seleccionamos como jurisdicción sobre la cual profundizar nuestra indagación. Esto nos permitió adentrarnos en la dinámica de trabajo del equipo de gestión provincial, así como también acercarnos y entrevistar a docentes de esa provincia que participaron de las capacitaciones que desarrolló allí El.

Desde la consideración de que este trabajo presenta algunos de los hallazgos de nuestra investigación, en esta ocasión recuperaremos distintos fragmentos de algunas de las entrevistas que resultan de interés para las temáticas aquí abordadas. Recuperamos diez entrevistas entre las cuales se encuentran las realizadas al equipo de Escuelas de Innovación, especialistas en el área, integrantes de la gestión educativa de Mendoza y docentes de dicha provincia. Para identificarlos a lo largo del texto los codificamos de la siguiente manera: Entrevista a la Coordinadora General de Escuelas de Innovación (ECGEI - 10/05/2018); Entrevista a la Coordinadora de área Ciencias Sociales de Escuelas de Innovación (ECCSEI - 1/12/2017); Entrevista a los capacitadores de área de Ciencias Sociales de Escuelas de Innovación (ECACSEI 1 - 25/10/2018, ECACSEI 2 - 25/10/2018, ECACSEI 3 - 24/10/2018); Entrevista al Especialista en Didáctica de las Ciencias Sociales (EEDCS 26/06/2019); Entrevista a la Capacitadora de la Dirección de Políticas de Integración Digital de Mendoza (ECDPIDM 1 - 30/07/2019); Entrevista a Docentes de Mendoza (EDM 1 - 1/08/2019, EDM 2 - 31/07/2019, EDM 3 - 31/07/2019).

\section{El área de Ciencias sociales de Escuelas de Innovación}

El área de trabajo de Ciencias Sociales se configuró como uno de los grupos disciplinares que hacia el interior de Escuelas de Innovación diseñaron las propuestas formativas. En función de nuestro interés por recuperar el proceso de diseño del plan, comenzamos este recorrido a partir de la descripción de las características del área y del proceso que dio lugar a su conformación. En ese marco, una de las primeras tareas que se propusieron desde la gestión de Escuelas de Innovación (EI) al momento de su creación fue la conformación de estos equipos de trabajo de las distintas áreas. La primera Coordinadora General de El describe ese proceso y su acercamiento a quien fue la Coordinadora del área de Ciencias Sociales:

"Ese primer año 2011, en el transcurso de dos meses entre febrero, marzo y principios de abril armamos los equipos de las áreas básicas. Yo la conocí a Ángeles porque habíamos 
trabajado juntas en el portal Educared [...]. Ella es profesora de Historia, ya tenía ese cruce entre el contenido y las tecnologías" (ECGEI - 10/05/2018).

Según la entrevistada, "fue difícil el armado de esos equipos porque los perfiles tenían que tener hecho el cruce" (ECGEI - 10/05/2018). Este "cruce" hace referencia a una doble expertise en el manejo de los medios digitales y el conocimiento de la disciplina del área, y buscó generar la articulación entre el conocimiento disciplinar de las Ciencias Sociales, el conocimiento didáctico y el conocimiento tecnológico (Mishra y Koehler, 2006).

En el caso de Ciencias Sociales la conformación del equipo de capacitadores estuvo a cargo de la Coordinadora del área, quien tomó esta tarea desde las posibilidades de reconocimiento y rastreo que le otorgaban su mirada y experiencia en el campo de las Ciencias Sociales. Según ella, lo que buscaban eran profesores que "trabajaran en escuelas secundarias prioritariamente y sino en la Universidad, y que tuvieran conocimiento y disposición para innovar en la enseñanza con las tecnologías digitales" (ECCSEI - 1/12/2017). De esta manera, se configuró un doble perfil sobre los integrantes del equipo de El que comprendía, por un lado, el conocimiento y el ejercicio activo de la enseñanza de Ciencias Sociales, preferentemente en el nivel secundario, y, por el otro, predisposición y conocimiento de la utilización de tecnologías.

Ambas cualidades eran reconocidas como parte del recorrido profesional y de la formación de la Coordinadora del área de Ciencias Sociales y configuró, como ya mencionamos, el perfil buscado posteriormente en los capacitadores que conformaron su equipo:

"La construcción de este equipo también para mí fue un logro. Creo que en ese sentido mi mayor contribución fue ese armado. Siento que como tengo una formación, por un lado, disciplinar y, por otro, en didáctica y tecnología educativa, eso ayudó a revisar algunas cuestiones que son distintas cuando uno solamente reconoce la mirada del especialista. Creo que el equipo aprendió de ese "mirar desde otro lugar". Y al mismo tiempo, esa mirada disciplinar y rigurosa que ellos aportaban también ayudó mucho a la construcción" (ECCSEI - 1/12/2017).

Resulta interesante recuperar de este relato la alusión a la construcción de una "mirada desde otro lugar" que no necesariamente representa la mirada únicamente del "especialista" en el conocimiento de la disciplina. La entrevistada reivindica esta característica central de la composición del equipo de capacitadores basada en un manejo del contenido disciplinar en vinculación con el uso de tecnologías. Una de las capacitadoras del área identificó que este aspecto no era resaltado únicamente por los equipos de coordinación, sino que también era muy bien recibido por parte de los docentes que participaban de las capacitaciones:

"Les gustaba mucho también que fuéramos capacitadores de Ciencias Sociales porque todos los docentes nos decían que todos los cursos de tecnología que habían tenido eran tecnólogos. La riqueza de poder trabajar didáctica de las Ciencias Sociales con tecnología ¿no?" (ECACSEl 2 - 25/10/2018).

Aquí aparece una perspectiva que desde El se buscó evitar, y es que las capacitaciones no fueran abordadas únicamente por expertos en la utilización de tecnologías. Es decir, no se 
buscaba únicamente especialistas en la disciplina ni especialistas en tecnologías, sino perfiles que pudieran entrecruzar ambas especialidades. En la propuesta de El, la formación de los docentes que ejercen en el nivel secundario se dio centrada en la disciplina en la cual se desempeñan, por lo que resulta comprensible una mayor cercanía y recepción de los docentes capacitados a una propuesta que recupera su formación de base y los contenidos que trabajan en el aula cotidianamente para desde allí realizar un abordaje con tecnologías.

Otro aspecto a considerar en la conformación del equipo de capacitación del área de Ciencias Sociales, tiene que ver con una atención puesta a la presencia entre sus integrantes de la formación en las distintas disciplinas que integran las Ciencias Sociales:

"Primero siempre tratamos de equilibrar en Ciencias Sociales así que teníamos gente de Historia en el equipo, gente de Geo, estaba después esta compañera de Artes y había trabajado con nosotros una compañera de Antropología que había hecho su investigación en documentales" (ECACSEI 3 - 24/10/2018).

La interdisciplinariedad en el marco de las Ciencias Sociales fue otra de las características de la conformación del equipo. Este interés por garantizar una composición interdisciplinaria por parte de El, coincide con la perspectiva brindada con el especialista en Didáctica de Ciencias Sociales entrevistado ya que según él, usualmente el profesorado recibe "una formación estrictamente disciplinar en el sentido de pensar los procesos sociales, históricos, culturales" cuando uno en realidad puede "enseñar Historia en diálogo con otras disciplinas del campo de las Ciencias Sociales o incluso inter o transdisciplinarmente, y ahí es cuando entiendo que uno va a la disciplina o vuelve a la disciplina con otras preguntas" (EEDCS - 26/06/2019). Por ejemplo, la composición interdisciplinar del equipo del área de Ciencias Sociales dio como resultado una articulación conjunta para el abordaje de las nociones temporales y espaciales:

"Hubo un trabajo también de enriquecimiento mucho desde las disciplinas, porque los que veníamos más desde lo espacial solemos trabajar más desde la actualidad, pero vivimos inmersos en esto de sumergirnos en el pasado, y de tratar que las estrategias de las miradas temporales estuvieran reforzadas aún en las secuencias que trabajábamos prioritariamente con una perspectiva espacial" (ECACSEl 1 - 25/10/2018).

De esta manera, el equipo de trabajo del área de Ciencias Sociales se conformó, a partir de una fuerte impronta de la formación de su coordinadora y su criterio de selección, con profesores que se caracterizaron por el ejercicio activo de la docencia y por su experiencia previa y apertura a la innovación con tecnologías. Este cruce basado en la articulación entre disciplina, enseñanza y tecnología, se combinó además con un equilibrio de selección que permitiera dotar al grupo de una interdisciplinariedad que integró las distintas disciplinas que conforman las Ciencias Sociales.

Con respecto a las tareas que el equipo de Ciencias Sociales tenía a su cargo, eran todas aquellas abocadas a la planificación y realización de las acciones de capacitación del área en las distintas jurisdicciones. Desde la selección de las temáticas y secuencias didácticas a trabajar hasta su puesta en práctica en las distintas escuelas del país. Según expresión de la Coordinadora del 
área, para resolver todas estas cuestiones se realizaban una serie de reuniones con las siguientes características:

"La lógica de funcionamiento que tuvimos en el grupo tenía una frecuencia de reuniones muy asidua, semanales. Eso nos permitía trabajar tanto en la selección de contenidos y estrategias de trabajo, como en nuestra propia formación, y abrirnos al uso de distintos recursos tecnológicos que cada uno conocía más o menos e íbamos estableciendo como un piso de homogeneidades, entre todo el grupo y/o investigando nuevos y poniendo en común esas nuevas opciones que encontrabas" (ECACSEl 1 - 25/10/2018).

Resulta interesante identificar cómo la intención de indagar y formarse de manera continua sobre las temáticas a trabajar conformó una de las características propias del trabajo del área, así como la continuidad de las reuniones del equipo. En estas reuniones el rol de la Coordinadora del área aparece como fundamental en las definiciones adoptadas:

"Siempre hacíamos reuniones en todo el equipo y capaz trabajábamos más de a dos para armar una propuesta, pero casi siempre se discutía entre todos, y la última palabra siempre la tomaba Ángeles, eso era así, pero siempre muy en diálogo" (ECACSEI 2 25/10/2018).

Según una de las capacitadoras, en función de cada tema "determinado grupo o personas en particular tenían que desarrollar más, para después volver a analizar y ver si eran viables, cómo iban tomando forma" (ECACSEI 1 - 25/10/2018). Estas reuniones configuraban la etapa de preparación de las capacitaciones "donde no estabas todavía implementando en terreno" y "el trabajo más fuerte era investigar, leer, proponer cosas, amasar un poco la cocina de lo que pensábamos hacer" (ECACSEI 1 - 25/10/2018).

En la dinámica de trabajo del área es posible identificar un momento preparatorio en donde las reuniones estaban abocadas a la formación sobre contenidos disciplinares, en la investigación sobre novedades tecnológicas y mediaciones digitales, y sobre el abordaje didáctico a realizar en las propuestas de capacitación. Todo esto con una dirección clara de la Coordinadora del área específicamente en la división de tareas y en las decisiones finales.

Pasado este primer momento de planificación y una vez que las capacitaciones se encontraban en marcha, en las reuniones semanales se incorporaba una segunda instancia entre los temas a abordar referidos a la reflexión y el balance posterior al trabajo en las jurisdicciones:

"Cuando ya estabas trabajando en terreno, la primera parte de esas reuniones tenía que ver con la puesta en común de lo que había pasado y a partir de ahí repensar si replicabas, si adaptabas, si había funcionado si no, como todo el tiempo poner en juego lo que pasó en la realidad, y repensar a partir de eso" (ECACSEI 1 - 25/10/2018).

La adaptación del trabajo de El a las jurisdicciones en las cuales realizaba sus capacitaciones era una de sus características específicas y formaba parte de un proceso general de articulación de la política de formación docente con la gestión educativa provincial. Podemos destacar que la adaptación de la propuesta de capacitación de acuerdo a las características, necesidades y experiencias de cada territorio formaba parte también del grupo de Ciencias 
Sociales y se convertía en uno de los temas centrales en las reuniones. En relación con la adaptación, la Coordinadora del área lo describe de la siguiente manera:

"No había un modelo único de capacitación, sino que se acordaba con cada jurisdicción el tipo de intervención que se iba a hacer considerando las características de los docentes y los cursos sobre los que se iba a trabajar" (ECCSEI - 1/12/2017).

La negociación con cada jurisdicción determinaba finalmente el formato de la propuesta que llevaría el plan a cada provincia, aspecto que se convertía en un desafío en tanto diversificaba la construcción de las propuestas en función de cada destinatario provincial. Esta situación generaba que en las reuniones de trabajo del grupo de Ciencias sociales tuvieran que adaptar también sus planificaciones y propuestas:

"Esto tenía que ver con qué distrito te tocaba, qué características tenía la propuesta en ese distrito, que por ahí podía haber una clase de más, una clase de menos o podías estar trabajando como destinatarios directamente con los docentes, en algún momento al principio fue con docentes y alumnos o con los equipos de formación territorial. Esto hacía que tuvieras que repensar y adaptar algunas cuestiones, por ejemplo, la adecuación de los contenidos a las características locales" (ECACSEI 1 - 25/10/2018).

De esta manera, se configuró un arduo trabajo de formación y planificación grupal previo que se mantuvo en reflexión constante a partir de la readaptación de las propuestas de capacitación de acuerdo a las demandas de cada jurisdicción. La intención de que la propuesta de El se convierta en replicable y sustentable conformaba una de sus características fundantes y en ese sentido la formación de formadores resultaba una de las acciones claves del plan. Por lo tanto, una de las modificaciones y adaptaciones que se realizaba a las propuestas tenía que ver con la variación de los destinatarios, ya que no se construía la misma propuesta para docentes que para cuando se trabajaba con formadores de formadores. Este aspecto fue señalado por dos de las capacitadoras del área al momento de ser entrevistadas:

"Eso era rediseñar todo el tiempo la propuesta porque no hablás de las mismas cosas con el que tenés que enseñarle o tratar de transmitir, de construir, las formas en las que se enseña, que cuando es la producción directa con el docente y entonces son hay un tema que es, que son los problemas en el aula, la coyuntura, como que son otras las variables que están en juego" (ECACSEI 3 - 24/10/2018).

"Por ejemplo, con ellos, mirábamos la misma secuencia, pero en vez de ejecutarla paso a paso como hacíamos con los docentes, teníamos que tener una mirada de meta análisis, de enseñarles a enseñar. Digamos que había otra perspectiva en cómo trabajar con esos docentes que tenían este otro rol de replicador" (ECACSEI 1 - 25/10/2018).

Además del desafío del rediseño de la propuesta en función de la demanda de la jurisdicción, la variación de los destinatarios que recibían la propuesta se convertía en otra de las cuestiones a abordar al momento de construir las planificaciones de manera previa.

Por último, en lo que refiere a la organización previa de las acciones de capacitación en los distritos, otro de los aspectos que tuvo que resolver el área de Ciencias Sociales fue el referido a la 
división de la realización de las capacitaciones y el sostenimiento de la relación con cada jurisdicción entre los integrantes del área de Ciencias Sociales de El. En palabras de la Coordinadora esto significó una gran dificultad como veremos a continuación:

"Fue muy difícil sostener la continuidad de los capacitadores en la misma jurisdicción.

Teníamos dos problemas, primero que las capacitaciones eran en simultáneo, entonces había que ver cómo armábamos la agenda para poder estar en tres lugares al mismo tiempo. Pero además teníamos otra dificultad adicional: la especificidad disciplinar dentro de la misma área. Yo quería que cuando trabajábamos temas que pivoteaban más en Historia, en Geografía o en Economía, el capacitador fuera especialista en el tema. Si bien todos estaban preparados para abordar todos los temas, nos interesaba mucho trabajar con el mayor nivel de expertise posible. Entonces eso sumaba complejidad a la asignación de capacitadores, porque el ciclo de formación tenía un recorrido a lo largo de esos cuatro o cinco encuentros con los docentes" (ECCSEI - 1/12/2017).

Ante este escenario se exploraron distintas alternativas que buscaban conciliar, por un lado, la presencia de la especificidad disciplinar en función de los temas que se trataran, y por el otro la continuidad de los capacitadores en la misma jurisdicción:

"Probamos dos criterios organizativos, uno era al principio, que cada uno, se hiciera cargo, en forma predominante de una o dos secuencias, y la tuviera aceitada así, y la llevara a todos lados. Eso tenía algo de bueno, pero cuando había que rotar uno no estaba tan empapado de las cosas que pasaban en la implementación de las otras, sabíamos de qué se trataban, cómo eran, digamos, pero no la habías vivido. En muchos ámbitos después fue difícil mantener esto, entonces priorizamos la otra cuestión, sobre todo en el trabajo en el interior, de ir, que por lo menos uno de los dos capacitadores se repitiera, para que también el colega, el docente, no se encontrara siempre con una cara nueva y se perdiera. Buscamos mantener por lo menos uno de los dos, y si era posible los dos, mejor. Eso parcialmente lo logramos al final" (ECACSEI 1 - 25/10/2018).

En este segundo esquema adoptado en el cual se repetían los capacitadores para sostener el contacto y el conocimiento por parte de los docentes de la jurisdicción también se buscaba que el grupo de capacitadores fuera equilibrado interdisciplinariamente:

"Para viajar combinaban alguien de geo y alguien de historia y tratábamos de sostener esa continuidad, como que no daba lo mismo, y bueno eventualmente venía otro compañero mío pero siempre había alguien que conocía a los docentes, que había entendido algo de la idiosincrasia de lo que pasaba ahí porque si no te perdéis un montón y por eso como que esta tensión entre lo particular y la posibilidad de escalar, porque la verdad que la particularidad pesa mucho, pero también te impide algo del hecho del masivo" (ECACSEI 3 - 24/10/2018).

Estos relatos nos permiten identificar tres criterios centrales que articularon la organización del desarrollo de las capacitaciones en las distintas provincias: la continuidad en la jurisdicción, el conocimiento de la propuesta didáctica y la interdisciplinariedad del equipo. Así, se 
conformó un esquema en donde se buscaba que quienes viajaban a la jurisdicción ya hubieran participado de capacitaciones previas allí, que conocieran y tuvieran experiencia en la propuesta que les tocaba llevar adelante y que haya presencia de más de una disciplina en la formación de los capacitadores.

Observar la dificultad que implicaba el armado de los grupos de capacitadores que viajaban a las distintas jurisdicciones nos remite algunos interrogantes con respecto al formato de la propuesta. Nos preguntamos en este caso si la formación de un equipo de trabajo con su pertenencia geográfica en la Ciudad Autónoma de Buenos Aires, desde donde viajaban a las distintas jurisdicciones a realizar las capacitaciones no actuaba en cierta manera cómo desventaja al momento de garantizar la continuidad en las jurisdicciones. Quizá la articulación con los equipos jurisdiccionales y la posibilidad de tener miembros de los equipos de Escuelas de Innovación en cada provincia podría haber abierto otras posibilidades al momento de organizar los dispositivos formativos en las distintas escuelas del país.

\section{La experiencia de los docentes capacitados}

Dentro de nuestra investigación profundizamos sobre la puesta en acto de Escuelas de Innovación (EI) en la provincia de Mendoza, fundamentalmente a través de las voces de los docentes participantes en las acciones propuestas por El. Consideramos que recuperar a modo de balance la vivencia que estos sujetos tuvieron en relación con el plan de capacitación que analizamos nos permite destacar algunas fortalezas de la propuesta de Ciencias Sociales de El. En líneas generales, los distintos relatos que veremos a continuación muestran una fuerte valoración por parte de los docentes de la propuesta de El tanto desde sus aspectos organizacionales como en sus perspectivas formativas didácticas, tecnológicas y sobre la enseñanza de las Ciencias Sociales.

Sobre la propuesta en sí misma, una de las docentes entrevistadas destacó la organización con que las instancias de capacitación de El se desarrollaron y la utilización del material didáctico producido por el plan:

"Estaba muy bien organizado, ellas estaban muy bien organizadas, como iban pautando las actividades, traían todo armado, porque la verdad que, si no funcionaba internet en ese momento, todo el material que ellas tenían lo pasábamos todo a través de la red de las redes ad hoc o de pendrive y empezábamos a trabajar. Estábamos desde las 9 de la mañana hasta las 5 de la tarde trabajando en una escuela, entonces lo bueno era que teníamos la liberación, y eso nos permitió estar todo el día capacitándonos y poder juntarnos con otros profes y compartir experiencias con otros profes, eso fue buenísimo, pero lo bueno es que está armado y todo lo que las chicas nos dejaron yo lo tengo y lo sigo reutilizando. Lo que mandaron las chicas, o sea, siempre va, bueno, voy a leer, y todo lo que ellas me hicieron leer, después yo lo tuve que leer para la capacitación de directivos y la verdad que no tiene desperdicio el material y lo sigo teniendo y si puedo lo comparto" (EDM 1 - 1/08/2019). 
Observamos aquí que tanto la organización del funcionamiento del encuentro de formación, así como la vivencia de la experiencia de compartir la instancia con otros colegas son aspectos resaltados por la docente entrevistada. Al mismo tiempo, considera como uno de los puntos altos de El el material entregado y producido por el plan, el cual continúa siendo material de consulta y compartido entre colegas. Esta producción didáctica tomó el formato de un ebook, el cual hemos analizado específicamente en un trabajo anterior:

"El equipo de Ciencias Sociales sintetizó en el ebook, cuatro propuestas didácticas con gran parte del trabajo realizado en las capacitaciones y encuentros de trabajo con docentes. Las propuestas buscaron construir entramados explicativos a partir del análisis de interpretaciones, conceptos y procesos. Los núcleos temáticos que dieron el marco a cada una de ellas fueron "La organización del dominio colonial europeo en América", "Nuevas identidades en la sociedad global", "Urbanización, desigualdad y segregación espacial", "Globalización y ordenamiento territorial: la transformación del espacio rural". Pensadas para ser abordadas en distintos años y asignaturas de la educación secundaria estas propuestas contenían además un eje transversal que las aglutinaba, la comprensión de los fenómenos económicos, sociales, políticos y culturales asociados al proceso de globalización. Cada propuesta se encontraba organizada a partir de una presentación general en donde se exponía la fundamentación disciplinar y didáctica desde la cual fue pensada, se establecían los vínculos con los contenidos curriculares (Núcleos de Aprendizaje Prioritarios) y se explicitaba el modo en que la tecnología podía enriquecer el abordaje de la temática seleccionada. Posteriormente, el desarrollo de la propuesta se componía de una secuencia de actividades, con sus respectivas consignas y recursos didácticos, y la propuesta evaluativa de los contenidos trabajados. Por último, la propuesta finalizaba con un metaanálisis donde se recuperaba didácticamente el sentido del abordaje realizado y con una bibliografía y webgrafía a modo de sugerencia para la preparación de clases" (Marchetti y Bazán, 2019, p.49).

El hecho de que este material producido por El, el cual se encuentra dado de baja en la web, continúe circulando entre los docentes nos habla de una persistencia de sus enseñanzas entre quienes participaron de las capacitaciones.

Por otro lado, la enseñanza del trabajo en equipo aparece como otro de los aspectos resaltados por los docentes participantes, fundamentalmente a partir de que no se encontraban habituados a realizarlo. Al mismo tiempo, la docente entrevistada reconoce la potencialidad de las tecnologías como facilitadoras para el encuentro:

"Nos enseñaron también a trabajar en equipo que nosotros no estábamos acostumbrados a eso y que el trabajar en equipo se puede hacer, aunque no sea de forma presencial, podíamos aprovechar conectarnos a través del Facebook, ponernos de acuerdo, y que te daba flexibilidad. A ver, la mentalidad que traíamos eran las reuniones de área que las peleábamos para que las dieran dentro del horario de clase y si no te tenías que juntar afuera y era todo un tema ponernos de acuerdo, en cambio acá vos podías entrar al 
Facebook en cualquier momento y si veías la intervención de un colega hace dos horas, bueno, vos podías intervenir en ese momento, era más flexible, y si no nos poníamos todos de acuerdo y chateabamos a la vez, pero no era necesario" (EDM 2 - 31/07/2019).

Así se destacan las posibilidades ofrecidas por El al crear grupos de facebook ${ }^{2}$ y ponerlos a disposición como instancias de socialización entre los colegas. El fomento al trabajo en equipo y la construcción de canales mediante los cuales realizar el intercambio y el encuentro aparecen también como dos aspectos a resaltar dentro de la propuesta de El.

En relación con la concepción didáctica sobre el uso de las tecnologías, una de las docentes resaltó como aprendizaje de las capacitaciones de El el hecho de realizar sus planificaciones con amplitud, sin encerrarse en una sola herramienta o estrategia y con mayor capacidad de apertura a las experiencias de los colegas:

"Me sirvió. Primero por entender que no hay que atarse a una herramienta o a una estrategia particular, atarte a una sola te estanca, yo siempre discuto y discutía antes y ahora con el rol de director también, de que hay que abrir la cabeza, abrir la mirada, y que escuchar las otras experiencias, cómo uno utilizó la TIC para determinada cuestión, o cómo utilizó una fotocopia para otra, me parece que me sirvió para abrir un poco más la cabeza y no atarse a una herramienta" (EDM 3 - 31/07/2019).

La importancia de generar una apertura a las múltiples opciones que ofrece el trabajo con tecnologías es resaltada por esta docente como aprendizaje de las capacitaciones y se encuentra en sintonía con los objetivos del plan orientados a que los docentes conozcan y experimenten el uso de tecnologías, y reflexionen sobre las oportunidades que éstas ofrecen.

La perspectiva sobre la enseñanza de las Ciencias Sociales que construyó Escuelas de Innovación fue otro de los aspectos que resaltaron dentro del relato de la experiencia de los docentes de Mendoza entrevistados. En primer lugar, se hace visible cierta ruptura respecto a la concepción de la enseñanza de la Historia con la que los docentes mendocinos se encontraban familiarizados:

"El plan Escuelas de Innovación traía como una propuesta, digamos, más superadora, la formación de los profesores de Historia, acá en Mendoza es una formación más, del dato, más del hecho, de lo enciclopédico, ¿se entiende? Trabajar la historia desde los principios explicativos, pensar en multiperspectividad, pensar en una multicausalidad.

Trabajar el tema de los problemas que era, eso te iba a decir, que fue la gran apuesta fuerte de Escuelas de Innovación, acá costó muchísimo, era mucho más natural, sigue siendo mucho más natural, la explicación del proceso, esto comienza acá, tiene estas características, estos son los actores sociales, mucho tampoco me meto con los actores sociales, ¿no?" (ECDPIDM 1 - 30/07/2019).

Frente a una formación asociada a una mirada más cercana a una concepción positivista de la Historia poniendo en un lugar central a los datos o los hechos históricos, según la entrevistada, la aparición de la multiperspectividad, la multicausalidad y la explicación de los procesos históricos a partir de los problemas sociales ampliaron las perspectivas vigentes. En esa 
sintonía, otro de los docentes recupera la importancia que significó para ellos el aprendizaje de la perspectiva de enseñanza de la Historia basada en problemas:

"Estábamos muy contentos porque siempre te plantean la historia como un problema.

Suponete empezaron con el proceso de colonización y lo planteaban como un problema a resolver, y una de las cosas que yo me acuerdo que lo sigo manteniendo es la importancia de la pregunta que le vas a hacer a los chicos o vos mismo te hacés para poder, diríamos a ver, para qué sirve lo que estamos aprendiendo" (EDM 1 - 1/08/2019).

El lugar de la pregunta dentro de esta perspectiva y la invitación a la reflexión sobre lo que se está enseñando y de qué le sirve ese aprendizaje, aparecen también como puntos destacados. Hemos visto que la pregunta resulta central para motorizar el conocimiento a la vez que permite producir nuevos dentro de un enfoque interdisciplinar que busca problematizar sobre la realidad social (Jara, 2020). Como podemos ver, la propuesta de El no sólo le acercó a los docentes lineamientos sobre los cuales incorporar tecnologías en sus clases, sino que en una fuerte conexión con la mirada disciplinar acercó nuevas formas de abordaje de la enseñanza de las Ciencias Sociales. Así, la asociación con los problemas del presente y el debate sobre el conocimiento histórico y su producción también formaron parte de los aspectos destacados por los docentes capacitados por El:

"Si, otra mirada, por ejemplo, lo de la provisoriedad del conocimiento histórico que es una construcción y que todo es provisorio porque la ciencia va avanzando, va realizando los paradigmas. En otras cosas me reafirmó, en esto de que cuando enseñamos Historia enseñamos bueno, la historia se construye desde el presente, y desde el presente miramos al pasado, eso fue para mí un enriquecimiento, fue como que me abrió así el panorama, las perspectivas" (EDM 2 - 31/07/2019).

De esta manera, podemos observar cómo la perspectiva construida por El para el área de Ciencias Sociales no generó un aporte únicamente en términos de abordar distintos recorridos sobre los cuales generar prácticas de enseñanza con medios digitales, sino que desde el plan se mantuvo una coherencia con su propuesta disciplinar de tal forma que los aportes para repensar el abordaje de los contenidos de Ciencias Sociales fueron recuperados por los docentes capacitados de la misma forma o más que las nociones trabajadas para la inclusión de tecnologías. Una de las docentes reflexionó en este sentido sobre el aporte de El y la conexión entre tecnologías y Ciencias Sociales:

"A varios profesores y profesoras les sirvió para tener otra mirada de las Ciencias Sociales. Me parece que sirvió mucho para eso, la tecnología fue una herramienta más de lo que sirvió para que muchos profesores y profesoras tuvieran otra mirada de la historia" (EDM 3 - 31/07/2019).

Sin dejar de lado su impronta de capacitar en el uso de las tecnologías para la enseñanza de las Ciencias Sociales, en las capacitaciones de El el rol de la tecnología como mediatizadora y como vía para potenciar y enriquecer las prácticas de enseñanza quedó claro en estos docentes, que en ningún momento perdieron de vista los aportes propuestos por el plan para repensar de 
manera conjunta su concepción de la enseñanza de las problemáticas sociales y su relación con el presente con la inclusión de tecnologías.

\section{Reflexiones finales}

El interés que planteamos al inicio de este artículo se centró en la recuperación de la experiencia del plan de capacitación docente Escuelas de Innovación (EI) a fin de destacar algunos aspectos que sirvan de insumo para los debates actuales y futuros sobre las propuestas formativas abocadas a la inclusión digital para la enseñanza de Ciencias Sociales en el nivel secundario. A modo de cierre, a continuación, destacamos aquellas características de El que consideramos posibles de ser retomadas en distintos dispositivos formativos.

En relación con la conformación del equipo de formadores destacamos la búsqueda de perfiles que posean, por un lado, el conocimiento y el ejercicio activo de la enseñanza de Ciencias Sociales y, por el otro, predisposición y conocimiento de la utilización de medios digitales. De la misma manera, la atención puesta en garantizar la presencia entre sus integrantes de perfiles provenientes de las distintas disciplinas que integran las Ciencias Sociales resulta otro aspecto a destacar en pos de abordar la complejidad que los estudios sociales posibilitan desde la interdisciplinariedad. Este grupo mantuvo además el interés por indagar y formarse de manera continua en las temáticas a trabajar en las propuestas formativas.

Con respecto a la dinámica de trabajo que adoptó el área de Ciencias Sociales de EI identificamos un primer momento de planificación en donde las reuniones estaban abocadas a la triple atención acerca de la formación sobre contenidos disciplinares, la investigación sobre novedades tecnológicas y mediaciones digitales, y sobre el abordaje didáctico a realizar en las propuestas formativas. En un segundo momento, en las reuniones semanales se daba lugar a la reflexión y el balance posterior sobre el trabajo realizado en cada provincia. De esta manera, se configuró un arduo trabajo de formación y planificación grupal previo que se mantuvo en reflexión constante a partir de la readaptación de las propuestas de capacitación de acuerdo a las demandas de cada jurisdicción. En este punto, destacamos fundamentalmente la rediscusión permanente en la que se encontraba la propuesta formativa en función de las experiencias que se tenían a partir del desarrollo de los distintos encuentros de capacitación en cada jurisdicción.

Sobre las características del grupo de formadores de El, hemos visto que su distribución para la realización de las capacitaciones en las distintas provincias del país se basó en tres criterios centrales: la continuidad del capacitador en la jurisdicción, el conocimiento de la propuesta didáctica y la interdisciplinariedad del equipo. Así, se conformó un esquema en donde se buscaba que quienes viajaban a la jurisdicción ya hubieran participado de capacitaciones previas allí, que conocieran y tuvieran experiencia en la propuesta que les tocaba llevar adelante y que haya presencia de más de una disciplina en la formación de los capacitadores. En este caso, señalamos también las dificultades en las que se encontraban en reiteradas ocasiones para cumplir los tres criterios mencionados. consideramos como un camino posible para continuar reflexionando sobre este tipo de dispositivos centrales que luego abarcan a la totalidad de las provincias, habilitar a la 
construcción de equipos con integrantes estables en las provincias que permitan garantizar la continuidad y el acompañamiento en sus jurisdicciones.

Por su parte, los relatos de los docentes nos permiten recuperar la potencia de los materiales didácticos elaborados por El para Ciencias Sociales, los cuales se han mantenido vigentes en tanto los docentes manifestaron la continuidad en su uso hasta cinco años después de finalizado el plan. La perspectiva didáctica de la inclusión de los medios digitales sobre las que se sustentaron las propuestas de El fue otro de los aspectos resaltados en las entrevistas de los docentes, junto con la mirada sobre el estudio de las Ciencias Sociales al incorporar la multiperspectividad, la multicausalidad y la explicación de los procesos históricos a partir de los problemas sociales como ejes de análisis.

Además de las cuestiones vinculadas a los contenidos disciplinares, los medios digitales y su enseñanza, el trabajo en equipo aparece como otro de los aspectos destacados por los docentes participantes, fundamentalmente a partir de que no se encontraban habituados a realizarlo. Si consideramos que una de las estrategias de El consistía en que los docentes capacitados pudieran compartir lo aprendido con sus colegas en las instituciones educativas el fomento al trabajo en equipo, al intercambio y al aprendizaje sobre la experiencia de otro docente se vuelven imprescindibles.

Consideramos que las características de Escuelas de Innovación que recuperamos en este artículo pueden resultar de utilidad al momento de diseñar nuevos dispositivos formativos. Ahora bien, estos debates en torno a la formación tendrán sentido si las políticas públicas formativas estatales son concebidas como un derecho de los docentes y se generan las condiciones para que pueda ser garantizadas. Para ello, destacamos en primer lugar, que será necesario que las perspectivas desde las cuales se integre a los medios digitales en la enseñanza de las distintas disciplinas continúen profundizándose en pos de articular las particularidades de cada disciplina con la riqueza de la interdisciplinariedad y los contenidos de cada área curricular. En segundo lugar, la articulación del trabajo colectivo entre colegas deberá contar con las liberaciones horarias y organizaciones curriculares necesarias para que dentro del horario escolar puedan generarse espacios de formación e intercambio que no resulten una sobrecarga laboral fuera de horario para los docentes. Por último, el acompañamiento a los docentes capacitados y el seguimiento sobre el trabajo realizado en sus clases luego de transitar por los encuentros formativos, se convierte en un desafío que permita que lo aprendido en esas instancias permee de manera directa en la transformación de las prácticas de enseñanza. 


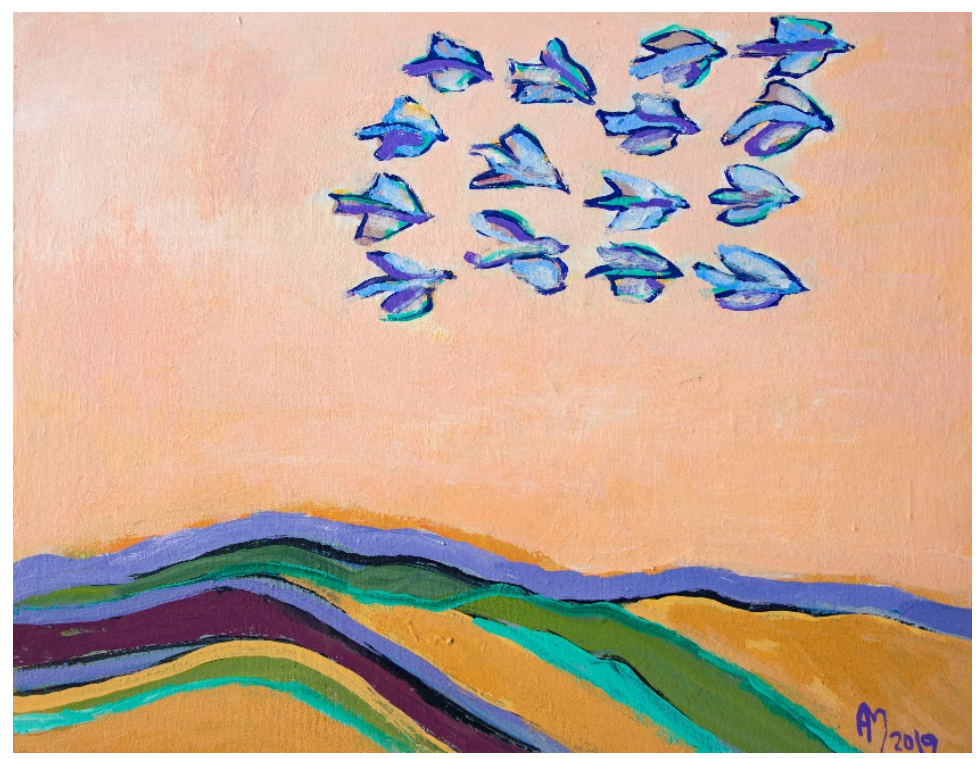

Volando juntos, acrílico al chapadur en tela. Ana María Martín

\section{Notas}

(1) "Las políticas públicas de formación docente continua en inclusión de medios digitales en Argentina. Un estudio interpretativo sobre la enseñanza de las Ciencias Sociales: el caso del plan Escuelas de Innovación (2011-2015). Directora: Dra. Sonia Bazán. Codirector: Dr. Luis Porta. Doctorado de Humanidades y Artes con mención en Ciencias de la Educación. Universidad Nacional de Rosario. Tesis presentada para defensa.

(2) Desde El intentaron sostener la conexión virtual entre encuentro y encuentro con los docentes a través de un campus virtual propio, aunque debido a dificultades con la utilización de esa plataforma se pasó en una segunda instancia a la utilización de grupos en Facebook que mejoraron las condiciones de participación e intercambio con los docentes capacitados.

\section{Bibliografía}

ANSES (2015). Escuelas de Innovación, 2011-2015. Buenos Aires: ANSES. Recuperado de https://issuu.com/escuelasdeinnovacion/docs/brief_escuelas_de_innovaci__n__2_ ANSES (2015a). Informe de gestión de Escuelas de Innovación: Logros y lecciones aprendidas 2011-2015. Buenos Aires: ANSES

Bolívar, A., Domingo, J., y Fernández, M. (2001). La investigación biográfico-narrativa en educación. Enfoque y metodología. Madrid: La Muralla.

Denzin, N. K. y Lincoln, Y. S. (2011). Introducción general. La investigación cualitativa como disciplina y como práctica. En Denzin, N. K. y Lincoln, Y. S. (Comps.), El campo de la investigación cualitativa. Manual de investigación cualitativa. Vol.I. Barcelona: Gedisa.

Jara, M. (2020). El enfoque interdisciplinar en la enseñanza de las Ciencias Sociales y Humanas. Reflexiones epistemológicas y metodológicas. Clío \& Asociados. La historia enseñada, (30), 75-89.

Marchetti, B. y Bazán, S. (2019). El plan de capacitación docente Escuelas de Innovación (2011-2015). Un estudio sobre el abordaje de la enseñanza de las Ciencias Sociales y la inclusión de tecnologías a través de las voces de sus protagonistas, Revista IRICEN 36, 33-56. 
Ministerio de Educación de la Nación (2015). La política educativa nacional 2003-2015. Inclusión y mejores aprendizajes para la igualdad educativa. Buenos Aires.

Mishra, P., \& Koehler, M. J. (2006). Technological Pedagogical Content Knowledge: A new framework for teacher knowledge. Teachers College Record, 108(6), 1017-1054.

Perczyk, J., Zapata, C., y Kisilevsky, M. (2011). Nuevas voces, nuevos escenarios: estudios evaluativos sobre el Programa Conectar Igualdad. Buenos Aires: Ministerio de Educación de la Nación.

Porta, L. y Flores, G. (2017). Investigación narrativa en educación: la expansión del valor biográfico. Revista del IICE, 41, 35-46.

Rapley, T. (2014). Los análisis de la conversación del discurso y de documentos en Investigación Cualitativa. Madrid: Morata.

Robles, B. (2011). La entrevista en profundidad: una técnica útil dentro del campo antropofísico. Cuicuilco, 18 (52), 39-49.

Soletic, A. (2015). Clase 13: Herramientas y Entornos Para El Aprendizaje de Las Ciencias Sociales. Diploma Superior Enseñanza de las Ciencias Sociales y de la Historia. FLACSO Virtual. https://dokumen.tips/documents/clase-13-herramientas-y-entornos-para-el-aprendizaje-de-las-cienciassociales.html 